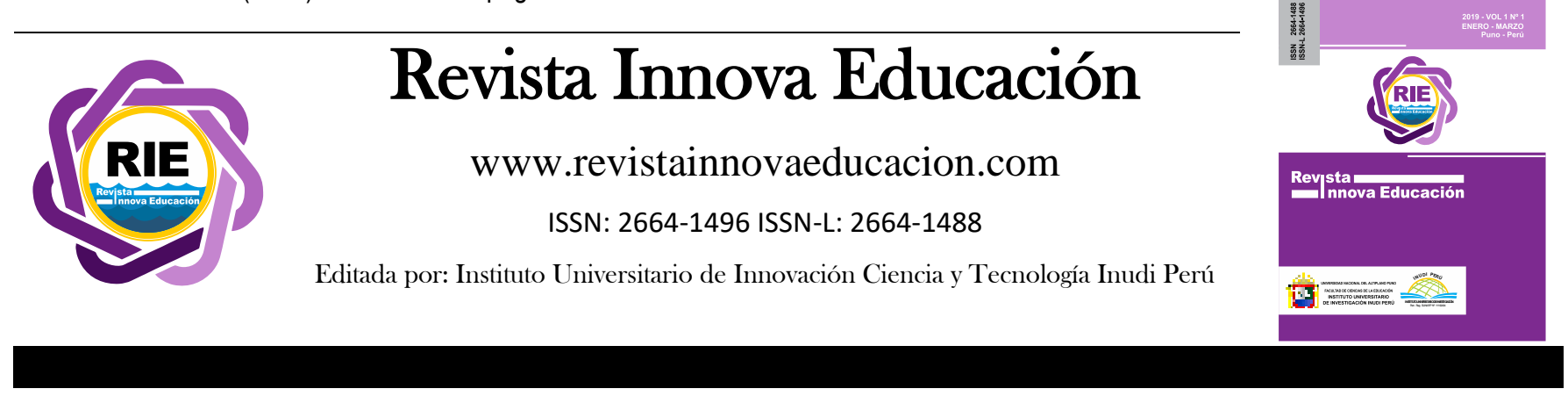

\title{
Escuelas de parentalidad positiva una estrategia pedagógica desde para las prácticas profesionales en las Ciencias Sociales
}

\author{
Schools of positive parenting a pedagogical strategy since for professional practices in the \\ Social Sciences
}

Darwin Cruz-García ${ }^{1 *}$

https://orcid.org/0000-0002-1858-1945

\author{
Jimena Ruiz* \\ https://orcid.org/0000-0002-5622-878X
}

DOI: https://doi.org/10.35622/j.rie.2020.01.009

*Corporación Universitaria Minuto de Dios, Antioquia - Antioquia, Colombia

Recibido 18/12/2019/ Aceptado 23/01/2020 Publicado 01/02/2020

\begin{tabular}{|c|c|}
\hline $\begin{array}{l}\text { ARTÍCULO ORIGINAL } \\
\text { PALABRAS CLAVE } \\
\text { Estrategia } \\
\text { pedagógica, } \\
\text { intervención social, } \\
\text { habilidades, } \\
\text { parentalidad positiva } \\
\text { y práctica. }\end{array}$ & $\begin{array}{l}\text { El trabajo plantea la reflexión de las escuelas de parentalidad positiva como estrategias } \\
\text { pedagógicas desde los escenarios de prácticas profesionales y establece la posibilidad } \\
\text { de la expresión de la intervención profesional, como proceso diverso; en donde, la } \\
\text { formación combina habilidad, teoría y práctica. Este estudio busca describir los } \\
\text { procesos pedagógicos a través de la utilización del método mixto en investigación. La } \\
\text { recolección de datos es a través de } 7 \text { entrevistas semiestructuradas a campos de práctica } \\
\text { profesional, } 25 \text { entrevistas a estudiantes en formación del programa de Trabajo Social, } \\
\text { y la implementación de } 2 \text { guías metodológicas dirigidas a población general y } \\
\text { funcionarios de institucionales en los municipios de Soacha y Sibaté. Por tanto, } \\
\text { fundamentar la pedagogía en lo social es necesario para contar distintos métodos que } \\
\text { permitan darle sentido y finalidad a la intervención profesional. En consecuencia, tratar } \\
\text { de comprender y explicar nuevas estrategias pedagógicas se convierte en una necesidad } \\
\text { fundamental para orientar intervenciones sociales. }\end{array}$ \\
\hline
\end{tabular}

\section{KEYWORDS}

Pedagogical strategy, social intervention, skills, positive parenting and practice.
The work raises the reflection of schools of positive parenting as pedagogical strategies from the scenarios of professional practices and establishes the possibility of expression of professional intervention, as a diverse process; where, training combines skill, theory and practice. This study seeks to describe the pedagogical processes using the mixed method in research. Data collection was given through 7 semi-structured interviews with fields of professional practice, 25 interviews with students in formation of the Social Work program, and the implementation of 2 methodological guides aimed at the general population and institutional officials in the municipalities of Soacha and Sibaté. Therefore, basing pedagogy on the social side is necessary to count different

\footnotetext{
${ }^{1}$ Correspondencia: darwin.cruz@uniminuto.edu.co
} 
methods that allow making sense and purpose for professional intervention. Accordingly, trying to understand and explain new pedagogical strategies becomes a fundamental need to guide social interventions.

\section{INTRODUCCIÓN}

El planteamiento de las escuelas de parentalidad positiva como estrategias pedagógicas innovadoras es de suma importancia para el desarrollo de competencias y habilidades en el quehacer profesional (Sanjurjo, 2012), en donde, los escenarios de prácticas profesionales son vitales para la formación (Fernández, 2005). Bajo esta premisa, recordamos a Luthans (2008) y Castellaro (2016) cuando evidencian cómo se orientan e implementan procesos que van moldeando formas de concebir la realidad, en espacios de instituciones para el reconocimiento de necesidades organizacionales y plantea la reflexión en el dominio de conocimientos teóricos y prácticos propios de las ciencias sociales.

Este ejercicio de formación constituye un referente según Villarroel y Bruna (2014) para el desarrollo de reflexiones en el ámbito universitario en temas pedagógicos y curriculares frente al impacto que genera las ciencias sociales en la región desde las familias como lo comparte Aguado-López (2014), ya que muchos de los hallazgos pueden ser de interés para la comunidad internacional, la mayoría abordan problemas peculiares de cada país, donde los medios locales son el canal natural y más próximo para difundir y debatir sus trabajos. Es decir, como la región requiere profesionales no sólo con el domino de ciertas técnicas y habilidades, sino también de competencias (Alarcón, R. 2002). Esta estrategia plantea un servicio de apoyo que desde la institución a las familias que contribuyen en condiciones para el desarrollo humano y personal de todos los componentes del sistema familiar.

Entonces, introduce planteamientos de Campanero (2018) en la identificación de necesidades, de Nistal (2008) en la planeación de intervenciones, de Vega de la Cruz (2016) en acciones de seguimiento y monitoreo como resultados desarrollados por medio de propuestas metodológicas que puedan generar cambios y transformaciones en las realidades sociales. Esto hace referencia a un conjunto de acciones organizadas y estructuradas que se plantean para intervenir problemas sociales y satisfacer necesidades (Llobet, 2012), por medio de una serie de actividades y tareas programadas con detalle y con una metodología de trabajo concreta destinadas a la consecución de un fin (Menéndez, S/F).

Por medio de habilidades de comunicación y liderazgo que faciliten el direccionamiento y la participación constante de la población en iniciativas de transformación (Sanjuro, 2012; 
Montenegro, 2017 \& Marchetti, 2018). De este modo, es importante la formación inicial que potencia la relación entre teoría y práctica para el análisis de situaciones institucionales y la comprensión de fenómenos sociales (Ibernón, 1998 \& Álvarez, 2012).

De allí la necesidad de trabajar estrategias institucionales que potencien las intervenciones formativas y que implica la participación no solo de la academia sino de organizaciones gubernamentales, no gubernamentales y organizaciones de base (Aran, 1999; Caride 2015 \& Ventosa, 2017). Es decir, aceptar los desafíos que se presentan en los diferentes campos de intervención profesional a partir de crear, procesar, adaptar, compartir y hacer accesible el conocimiento para mejorar los procedimientos en intervención a partir de la articulación institucional (Tobón, 2013 \& Hernández, 2016).

El articulo busca como objetivo describir los procesos pedagógicos en la formación de Trabajadores Sociales a partir de intervenciones institucionales en los escenarios de práctica profesional que permitan visibilizar según Solar (2009) la utilidad del aprendizaje en función del bienestar social y el reconocimiento como agentes que promueven procesos comunitarios (González, 2011 \& Solís, 2015). Esto permite plantear como pregunta de investigación ¿Cómo promueven las estrategias pedagógicas el desarrollo de habilidades formativas para intervenir realidades sociales?

Es relevante destacar como desde la revisión teórica se plantean modelos pedagógicos para la formación en ciencias sociales, que sustentan cambios en la reflexión de los nuevos retos que afrontan el quehacer profesional (Álvarez, 2012; Espinosa, 2016 \& Martínez, 2019). Por tanto, se plantean los siguientes enunciados desde diferentes autores que argumentan y ponen en discusión la concepción de intervención desde las estrategias pedagógicas.

Peña (2010) implica en los escenarios de intervención el reconocimiento de una capacidad técnica para responder a las demandas sociales y en Moreno (2018) frente a la concreción de acciones en lo cotidiano. Esta capacidad técnica estaría dada por las instituciones educativas, que se expresa en programas y currículos; que se manifiestan en las habilidades para el desarrollo de proyectos sociales (Murcia, 2008 \&Villamar, 2018).

De este modo, Ospina (2010) \& Frías (2010) plantean la importancia de fortalecer procesos pedagógicos referente la intervención profesional, en términos epistemológicos, teóricoconceptuales, metodológicos y ético-políticos, puesto que los espacios sociales que se dan en cualquier ámbito constituyen a asumir cada rol de acuerdo a las acciones planteadas y los contextos particulares frente a los escenarios de acción (De la Rúa, 2004; Rey, 2008 \& Rossato, 2013).

Por otra parte, Forero (2007) reconoce que los métodos caracterizan una profesión y su relación con propósitos y Moraleda (1998) plantea esta relación con estrategias sociales. Entonces, 
la pedagogía en la intervención plantea la necesidad de no imponer un afirmación o dogma si no se plantea como instrumento de análisis y reflexión del mismo que hacer profesional. Es decir, la intervención fomenta el desarrollo de grupos activos que intervienen y participan en el desarrollo de su entorno (Méndez, 2012 \& Moreno, 2018).

De esta manera, Baumman (1997) \& Falleti (2014) motivan el desarrollo de nuevas capacidades y cualidades, haciendo necesario soluciones innovadoras pertinentes para pensar la formación en ciencias sociales en los escenarios de prácticas profesionales (Rodríguez, Ramírez \& Betancur, 2016). De este modo, la intervención social metodológica debe incorporar controles recíprocos, donde los distintos miembros de un mismo equipo subsumen datos bajo categorías, objetivando la decisión en cuanto a su intervención y comparando resultados (Adorno, 2001 \& Nistal, 2008).

Para Miyamoto (2015) \& Silva (2015) las intervenciones sociales en relación a las emociones pueden jugar un papel importante en mejorar eficazmente una respuesta a una necesidad, reduciendo la brecha educativa en lo que existe en la teoría frente a la realidad social (Álvarez, 2012). Esto genera el desarrollo de habilidades formativas con la capacidad de respuesta a los procesos de enseñanza y aprendizaje (Sanjurjo, 2012).

Por tanto, las estrategias pedagógicas son construcciones alternativas que llevan a mejorar las intervenciones en ciencias sociales (Caride \& Caballo, 2015), y que tienen que ver con el rompimiento de paradigmas tradicionales sobre lo que se exige la intervención social (Barkin, 2012). Esto quiere decir, básicamente, que existen elementos en los escenarios de práctica profesional que se han evaluado como riesgosos para la intervención (Vallejo, 2012), una alternativa de otra manera de dar respuesta a las problemáticas sociales, puede ser útil para avanzar en términos de pedagogía (Gómez, 2016).

\section{MÉTODO}

Este estudio es de carácter descriptivo, presenta la utilización del método mixto en investigación, emprende puntos de interés en el campo de la pedagogía en las ciencias sociales (Morales, 2010). Es una revisión acerca de los procesos de intervención social en los municipios de Soacha y Sibate, con la necesidad de comprender las propuestas pedagógicas que aportan a las necesidades del contexto (Estrada, 2009 \& Noguera, 2014).

El enfoque utilizado en esta investigación es el histórico-hermenéutico según López y Hiader (2001) plantean que la compresión del tema se logra y se construye desde las relaciones contextuales e históricas de intervención en ciencias sociales, para este en particular en Soacha y 
Sibaté; según Gil (2011) por medio de este enfoque se busca comprender el quehacer, indagar situaciones, contextos, particularidades, simbologias, imaginarios, significaciones, persepciones, narrativas, conmovisiones, sentidos, esteticas, motivaciones, intenciones que se configuran en la vida cotidiana del hombre (Torres, 2012). Esto fue pertinente ya que permite identificar las habilidades desarrolladas en la formacion a través de los procesos pedagogicos en los escenarios de práctica profesional.

\section{Participantes}

Para la recolección de datos se encuesto a 25 estudiantes del programa de Trabajo Scoail de quinto y sexto semestre, mayores de 18 años, que tuvieron la experiencia de la practica profesional. Se seleccionaron de manera aleatoria en el marco de la implementación de la guía a población general en los campos de práctica profesional.

Esto se cruza con la percepción de 7 funcionarios institucionales que hacen parte del acompañamiento de la práctica, que fueron seleccionados según: Tiempo del campo, escenarios de implementación de la guía y viabilidad del estudio. En este sentido el proceso de recolección de la información es dado con el aval institucional y apoyo de los diferentes campos de práctica profesional, lideres sociales y estudiantes del programa.

\section{Estrategias de recopilación de la información}

De este modo, metodológicamente se desarrolló la recolección de datos a través de 7 entrevistas semiestructuradas a campos de práctica profesional, 25 entrevistas semiestructuradas a estudiantes en formación del programa de Trabajo Social en los municipios de Soacha y Sibaté; y, finalmente la implementación de 2 guías metodológicas, dirigidas a población general y funcionarios de las diferentes instituciones.

\section{Procedimiento para el análisis de la información}

Ejercicio que permitió conocer las percepciones frente a la construcción de estrategias pedagógicas en las intervenciones sociales en los escenarios de práctica profesional en Soacha y Sibate, en relación con la estrategia pedagógica, intervención social, habilidades y formación. Estas categorías de análisis orientan el proceso de organización, sistematización, análisis e interpretación de las estrategias pedagógicas y la comprensión de las intervenciones sociales.

Por tanto, esta propuesta metodológica se soporta en la necesidad de reflexionar los modelos pedagógicos como ejes de concepción de enseñanzas significativas basada en la auto- 
reflexión y comprensión de la propia realidad. De este modo, se realiza una lectura de los actores y el contexto, para identificar las diversas condiciones y necesidades del territorio de los municipios de Soacha y Sibaté.

\section{RESULTADOS}

Precisamente, la investigación logró comprender las estrategias pedagógicas a partir de guías para la intervención en ciencias sociales en el territorio (Campanero, 2018), esto permitió según Moreno (2018) el reconocimiento del contexto a través de la implementación de la mediación social, promoviendo en un 35\% la escucha empática, en un 55\% la comunicación constructiva, en un $15 \%$ proponer soluciones y en un $5 \%$ enfocarse en el futuro. El uso de esta condición facilito la lectura de necesidades trascendiendo en los espacios de práctica profesional (Murcia, 2008).

Entender las estrategias pedagógicas planteó la reflexión de las relaciones al interior de 105 familias en las 16 instituciones donde se implementó la estrategia (Caride \& Caballo, 2015), ya que esta plantea la necesidad de abrir la educación a la vida, la educación de todos y para todos. Esto direcciona el proceso de intervención del estudiante según Miyamoto (2015) en intentar comprender las necesidades y problemáticas en los escenarios de práctica profesional. Esto se argumenta, cuando el $65 \%$ reconoce la importancia de la intervención basada en el respeto, afecto y la comunicación (Rodríguez \& Restrepo, 2016); y un 55\% de los estudiantes manifiestan la importancia de la intervención para fortalecer la responsabilidad y garantizar los derechos (Villarroel, 2014 \& Ventosa, 2017).

Entonces, las estrategias pedagógicas para las ciencias sociales se encuentran relacionadas, según Moreno \& Molina (2018), con fortalecer la acción profesional orientada hacia la calidad y generar procesos de gestión de calidad eficaces y eficientes en el desarrollo comunitario. El estudio evidenció, de acuerdo a Rodríguez (2008), que las instituciones reconocen la importancia de la intervención en un 65\% para la sensibilización, en un 20\% para la apropiación y un 15\% para el autocuidado, que contribuyan a incrementar su calidad de vida y establecer procesos de comunicación basados en la calidez del trato, la atención integral, la empatía y autenticidad (Expósito, 2011, Rodríguez \& Restrepo, 2016).

De este modo, es importante señalar, según Moraleda (1998), que en los escenarios se identifica la empatía y habilidades comunicativas de los estudiantes como adecuadas en un $80 \%$, haciendo uso de un lenguaje esperanzador y generador que hizo posible la creación de nuevas estrategias para su propio proceso de formación personal y profesional (Silva, 2015). Esto planteó el desarrollo de un conjunto de capacidades que permitieron relacionarse de manera adecuada en 
cada contexto, expresando sentimientos, opiniones, deseos o necesidades (Vallejos 2012 \& Collado, 2014).

Por otro lado, un $20 \%$ de los escenarios esbozó la necesidad de fortalecer en habilidades de liderazgo a los estudiantes para abordar los diferentes contextos (Villarroel, 2014 \& Ventosa, 2017).

Esto relacionado al temor de los estudiantes de llevar a la practica los conocimientos aprendidos en la academia (Solis, 2015), visibilizando la necesidad de generar alternativas de apoyo que fomenten el desarrollo integral de todos los estudiantes con apoyo del contexto familiar, comunitario, laboral, escolar (Sluzki, 1996 \& Solar, 2009).

Esto plantea que el proceso pedagógico en Soacha y Sibaté es movilizador (Peña, 2010), ya que el $80 \%$ de la población fomento estrategias de participación al interior de las instituciones a partir de las intervenciones de los estudiantes incrementando el interés de acceder a los servicios institucionales (Murcia, 2008 \& Moreno, 2018). Estos encuentran formas distintas de orientación para la utilización de métodos de respuesta a sus necesidades como la autogestión (Rodríguez, 2016).

De este modo, según Gómez (2016) señala que la universidad juega un papel importante para potenciar las habilidades de los estudiantes a partir según González, López \& Collao (2011) en el desarrollo de estrategias pedagógicas en el tratamiento de intervenciones en campo, esto se respalda cuando el $96 \%$ de los escenarios plantean la importancia de la innovación en el acompañamiento pedagógico en los escenarios de práctica profesional (López \& Hiader, 2001). Esto traza un reto a nivel institucional que plantea la necesidad de un acompañamiento más estructurado a las necesidades de los estudiantes en los diferentes campos de práctica (Murcia, Martínez \& Ortiz, 2008).

Es así como las intervenciones de los estudiantes permitieron en un $40 \%$ la participación y convivencia en entornos protectores e influencia de valores (Noguera, 2014), en un 35\% prevención de violencias, y finalmente $25 \%$ en acciones afirmativas en contexto (Ospina, 2010). Estos procesos de intervención social aportaron a la construcción de comunidad y a dar respuesta las necesidades sociales de las familias (Campanero, 2018).

Es decir, que una estrategia pedagógica puede desarrollar y fortalecer competencias según Fernández (2005) para articular aspectos teóricos y prácticos en el quehacer profesional de acuerdo a las experiencias vividas en su entorno (González, Montenegro, López, Munita y Collao, 2011). Esto permitió incrementar y fomentar la rigurosidad en un $75 \%$ de su contenido, mejorar el entorno de aprendizajes adquiridos en un $25 \%$ en el proceso de formación, para compartir conocimiento 
dentro de las disciplinas y comprometer al profesorado en una activa e innovadora comunidad de práctica (Rodríguez, 2008).

En consecuencia, la construcción de estrategias pedagógicas para los escenarios de práctica profesional (Estrada, 2009), está sujeta a las relaciones establecidas con los estudiantes e instituciones (Noguera, Martí \& Almerich, 2014); en donde, se puede establecer como una condición resultante del desarrollo del aprendizaje mediante procesos de socialización y acompañamiento (Sanjuro, 2012), esto se soporta cuando el 49,9\% de los estudiantes manifiestan la importancia del rol docente para su acompañamiento en campo, el 20,5\% a la necesidad de plantear técnicas que les facilite la articulación teórico-práctica, el 15\% lo asociaron a la apertura del campo de práctica y el $10 \%$ a la capacidad individual para desenvolverse en escenarios comunitarios.

Por otra parte, las habilidades y la formación son expresiones en la intervención de cierto modo dadas en la academia a partir del currículo (Collado, 2014 \& Miyamoto, 2015). No obstante, también se constituye la intervención en una construcción desde la autonomía personal basada en las herramientas del entorno (Moreno \& Molina, 2018). Esto se establece cuando el 68,5\% de los estudiantes se siente cómodo en la implementación de las guías dadas para la intervención, o cuando el 72,5\% de los estudiantes manifiestan un apoyo y respaldo en los diferentes escenarios de práctica profesional (Noguera, 2014).

En esta medida, las intervenciones en ciencias sociales en Soacha y Sibaté se pueden encontrar en una ambivalencia frente a su identidad en la academia (Villamar \& Zambrano 2018) que va entre lo que le ha sido impuesto al estudiante (Tobón, 2013) y su posibilidad de construcción personal para la transformación social (Rossato, 2013).

Es así que las guías como estrategias pedagógicas en escenarios de prácticas profesionales en el territorio son concebidas en un $65 \%$ como un buen instrumento para la intervención (Expósito, 2011), un 15\% como guía para el rol profesional (Forero, 2007), 13\% la asocia a creencias e ideas de lo que es ser un Trabajador Social (Peña, 2010), 7\% no le da importancia. Esta diversidad, de concebir esta estrategia pedagógica se amplía en Soacha y Sibaté debido a características del contexto, como el encuentro de gran diversidad de personas, procedencias, culturas y etnias debido a fenómenos asociados por ejemplo al conflicto armado y el desplazamiento (De la Rúa, 2004).

Por tanto, las nuevas estrategias pedagógicas son alternativas que llevan a cabo los programas de ciencias sociales para la construcción de intervenciones (Ospina, 2010), y se relaciona con el rompimiento de paradigmas (Barkin, 2012) sobre lo que se exige la intervención profesional y su articulación con la teoría (Álvarez, 2012). Esto quiere decir, básicamente, que 
existen elementos de la academia y de la identidad propia según Silva (2015) que se han evaluado como relevantes para la transformación social, una alternativa de otra manera de hacer intervención para avanzar en el reconocimiento de los diferentes contextos.

\section{DISCUSIÓN}

Esto plantea según Expósito (2011) una reflexión acerca de la construcción de estrategias pedagógicas en los escenarios de prácticas profesionales y en la manera que se reconoce la formación en ciencias sociales de acuerdo a su contexto, incluyendo aquí la autopercepción y a los escenarios de práctica profesional (Rodríguez, Ramírez \& Restrepo, 2016), apropiando funciones, roles y papeles que fortalecen forma de intervenir en un contexto (Moreno \& Molina 2018).

De acuerdo con lo anterior y ciñéndonos estrictamente a este modelo, podemos preguntarnos entonces: ¿Cómo las estrategias pedagógicas fortalecen el perfil profesional en las ciencias sociales para la intervención en escenarios sociales? Si a través, de la aplicación de técnicas enfocadas filosófica e ideológicamente (Sanjurjo, 2012), que permiten coadyuvar a los procesos de cambios (Solar \& Díaz, 2009), para sustentar acciones enfocadas a determinados objetivos, conformándolos o negándolos (Ander-Egg, 1986).

Por otra parte, los métodos y propuestas según Forero (2007) aportan a la construcción de la intervención como proceso que articula e interpreta las realidades sociales, para distinguir aquello que es necesario transformar (Falleti, 2014). La reconstrucción al direccionar la acción, es una puesta hacia el futuro, (Kisnerman, 1998, retomado de Forero, 2007). Puesto que esto infiere en el avance metodológico de los diferentes problemas sociales que se pueden dar en los escenarios de práctica profesional (Menéndez, 2012).

En consonancia con lo anterior, el procedimiento de la intervención se debe centrar en el procedimiento académico que direcciona la relación entre lo ya aprendido con aquello por aprender, (Solano, 2007) Por ello es importante que las experiencias vividas de los estudiantes en los campos de práctica posean una enseñanza significativa, ya que permite identificar la utilidad del aprendizaje (Rossato \& Martínez, 2013).

Por tanto, las estrategias pedagógicas en intervención reconocen el papel de la formación y los vacíos de conocimiento (Silva, 2015), desarrollando competencias según Abadía (2015) comunicacionales, de relación interpersonal y metodológicas. En donde, las experiencias de los estudiantes generan un proceso de identidad a partir de subjetividades (Rey, 2008) e interacciones al interior de los campos, que mantienen las expectativas en el aprendizaje continuo del proceso de intervención (Solís, 2015). 
Esto significa que favorecen intereses particulares en tratar de comprender la realidad e innovar formas nuevas de interpretar problemas sociales (Barkin, 2012), ofreciendo la posibilidad de integrar y sintetizar los conocimientos (Fernández, 2015) y habilidades (Collado, 2014) adquiridas en su formación en campos institucionales a través de proyectos articulados e interdisciplinarios (Silva, 2015).

En este sentido, se puede señalar la importancia de la teoría y de la practica en el abordaje de las realidades sociales existentes, cuyo objetivo fundamental es generar procesos de intervención innovadores de alto impacto social (Álvarez, 2012).

\section{CONCLUSIONES}

En consecuencia, los docentes deben tener la capacidad para aplicar ajustes a estructuras metodológicas planteadas en la revisión que se desarrolla en los procesos autónomos de enseñanza y aprendizaje, ampliando procesos de organización y replanteamiento de ejes temáticos que permitan fortalecer la formación de los estudiantes en la intervención más contundente a las necesidades sociales y a las exigencias del entorno en el ámbito laboral, social y comunitario.

Finalmente, las estrategias pedagógicas contribuyen a la intervención profesional se piensa desde una práctica orientada al aprendizaje humano y a las representaciones sociales para la intervención de las ciencias sociales. En donde, los proyectos sociales que son iniciativas trascienden a la comprensión de métodos y procesos en educación social que posibilitan el empoderamiento de la población para la transformación de sus realidades sociales en relación a la construcción de tejido social.

Por tanto, el asumir la intervención social como un campo de análisis y de acción interdisciplinaria significa construir desde las necesidades o demandas presentadas en el contexto. Entonces, debe entenderse como campo de acción y de construcción que fundamenta el desarrollo de habilidades y establece nuevos desafíos en el quehacer profesional.

Por consiguiente, tratar de comprender y explicar las estrategias pedagógicas en los escenarios de práctica profesional se convierte en una necesidad fundamental para la transformación de dinámicas en Soacha y Sibate. Esto plantea el rompimiento de paradigmas tradicionales sobre lo que se exige que debe ser la intervención, puede ser útil para avanzar en términos de cambios sociales y reconocimiento institucional, deja claro que no existe una sola forma de hacer intervención y por lo tanto no existe una única forma de ver las realidades sociales. 


\section{REFERENCIAS BIBLIOGRÁFICAS}

Abadía Valle, A., Bueno García, C., Ubieto-Artur, M., Márquez Cebrián, M., Sabaté Díaz, S., Jorba Noguera, H., \& Pagès Costa, T. (2015). Competencias del buen docente universitario. Opinión de los estudiantes. REDU-Revista de Docencia Universitaria, 363 390.

Adorno, T. (2001). Epistemología y ciencias sociales. Ediciones Cátedra.

Aguado-López, E., Becerril-García, A., Arriola, M. L., \& Martínez-Domínguez, N. D. (2014). Iberoamérica en la ciencia de corriente principal (Thomson Reuters/Scopus): una región fragmentada. Interciencia, 39(8), 570-579.

Alarcón, R. (2002). La formación para el trabajo y el paradigma de formación por competencias. Calidad en la Educación, (16), 143-156.

Álvarez, C. Á. (2012). La relación teoría-práctica en los procesos de enseñanza-aprendizaje. Educatio Siglo XXI, 30(2), 383-402.

Ander-Egg, E. (1986). Diccionario de Trabajo Social. Bogota: Ed. El cd editor.

Aran, A. P. (1999). Didáctica en la educación social: Enseñar y aprender fuera de la escuela (Vol. 135). Graó.

Baumann, Z. (1999). Guerras por el espacio: informe de una carrera. La globalización. Consecuencias humanas.

Barkin, D. (2012). Hacia un nuevo paradigma social. Polis. Revista Latinoamericana, (33).

Campanero, M. P. P. (2018). Cómo detectar las necesidades de intervención socioeducativa (Vol. 21). Narcea Ediciones.

Caride, J. A., Gradaílle, R., \& Caballo, M. B. (2015). De la pedagogía social como educación, a la educación social como Pedagogía. Perfiles educativos, 37(148), 04-11.

Castellaro, M., Zanitti, L., \& Zingaretti, L. (2016). Una experiencia curricular de innovación y emprendedorismo en ingeniería.

Collado, E. D. (2014). Habilidades Sociales . Sociedad Española para el estudio de la Ansiedad y el Estrés (SEAS), 120-150 
De la Rúa, A. D. F. (2004). Los espacios sociales de la transnacionalidad. Una tipología de la integración relacional de los migrantes. Araucaria, 6(12).

Estrada, M. R. B., \& Martín, M. M. (2009). Educación en valores y educación emocional: propuestas para la acción pedagógica. Teoría de la Educación. Educación y Cultura en la Sociedad de la Información, 10(2), 263-275.

Espinosa Ríos, E. A. (2016). La reflexión y la mediación didáctica como parte fundamental en la enseñanza de las ciencias: un caso particular en los procesos de la formación docente. Tecné, Episteme y Didaxis: TED, (40), 175-209.

Exposito, C. B. (2011). Buenas Practicasde calidad y Trabajo Social . Alternativas, ISSN 1133$0473,57-74$.

Falleti, V. F. (2014). Las trasformaciones sociales:Una discusión sobre el cuerpo , la figura estatal y la Inclusión . Revista de Relaciones Internacionales, Estrategia y Seguridad vol. 9, núm. 2., 117,121 .

Fernández, J. T., \& Gámez, A. N. (2005). El desarrollo y la gestión de competencias profesionales: una mirada desde la formación. Revista Iberoamericana de educación, 37(2), 4.

Forero, N. A. (2007). Metodología, método y propuestas metodológicas en Trabajo Social. Revista Tendencia \& Retos $\mathrm{N}^{\circ} 12,122,126$.

Frías, J. D. L. C. T. (2006). Los procesos de formación de investigadores educativos: un acercamiento a su comprensión. Educatio 2. Revista Regional de Investigación Educativa.

Gómez, J. A. C. (2016). La pedagogía social en el diálogo de las universidades con la educación popular y la educación social. Revista Interamericana de Educación de Adultos, 38(1), 85106.

González, C., Montenegro, H., López, L., Munita, I., \& Collao, P. (2011). Relación entre la experiencia de aprendizaje de estudiantes universitarios y la docencia de sus profesores. Calidad en la Educación, (35), 21-49.

Hernández-Mosqueda, J. S., Tobón-Tobón, S., \& Guerrero-Rosas, G. (2016). Hacia una evaluación integral del desempeño: las rúbricas socioformativas. Ra Ximhai, 12(6), 359376. 
Imbernón, F. (1998) La formación y el desarrollo profesional del profesorado. Hacia una nueva cultura profesional. Barcelona: Graó

Llobet, M. P., Mateu, P. S., \& Ávila, N. R. (2012). Necesidades humanas: evolución del concepto según la perspectiva social. Aposta. Revista de Ciencias Sociales, (54), 1-12.

López, H., \& Hiader, J. (2001). Investigación cualitativa y participativa: un enfoque históricohermenéutico y crítico-social en Psicología y educación ambiental. Recuperado de: http://creativecommons. org/licenses/by-nc-sa/2.5/co/deed. es.

Luthans, F. (2008). Comportamiento organizacional. McGraw-Hill.

Marchetti, B. (2018). Un necesario recorrido desde la formación hacia la educación docente. Diálogos entre políticas públicas e inclusión digital. Entramados: educación y sociedad, (5), 47-57.

Martínez, M. I., Miralles, M. M., \& Cabezas, I. L. (2019). Repensando la formación teórica a través del prácticum: experiencias de una docente novel. Contextos educativos: Revista de educación, (23), 49-64.

Menéndez, S. L. (2012). Metodologia de la Intevención Social. Editorial Sintesis S,A, 22,23.

Miyamoto, K. (2015). Habilidades para el progreso social: el poder de las habilidades sociales y emocionales. Recuperado de https://www.oecd.org/edu/ceri/skills-for-socil-progressexecutive-summary.pdf.

Montenegro Núñez, J., Ramírez Celada, J., Schlicker Castaños, A., \& Solano Mena, D. (2017). Estado actual de la enseñanza de la psicología en Costa Rica.

Moraleda, M., Galán, A. G., \& Pinto, J. G. G. (1998). AECS, actitudes y estrategias cognitivas sociales. Madrid: Tea.

Morales, F. (2010). Tipos de investigación. Bogotá DC.

Moreno Camacho, M. A., \& Molina Valencia, N. (2018). La Intervención Social como Objeto de Estudio: Discursos, prácticas, problematizaciones y propuestas. Athenea digital: revista de pensamiento e investigación social, 18(3), 0007.

Murcia, D. M., Martínez, B. D., \& Ortíz, J. L. (2008). Intervención de Trabajo Social desde la práctica de entrenamiento profesional en instituciones educativas. Facultad de Trabajo Social, Universidad de La Salle 2000-2007. Bogotá., Colombia: Universidad de la Salle. 
Nistal, T. A. (2008). IAP, redes y mapas sociales: desde la investigación a la intervención social. Portularia: Revista de Trabajo Social, (8), 131-151.

Noguera, J. J. M., Martí-Vilar, M., \& Almerich, G. (2014). Responsabilidad social universitaria: influencia de valores y empatía en la autoatribución de comportamientos socialmente responsables. Revista latinoamericana de psicología, 46(3), 160-168.

Ospina, V. M. (2010). Resignificar la formación académica y la intervención profesional en lo social. Revista perspectivas Universidad del Valle , 56,57.

Peña, C. B. (2010). Intervención social desde el Trabajo Social: un campo de fuerzas en pugna. Revista perspectiva Universidad del Valle, , 49,68.

Rey, F. G. (2008). Subjetividad social, sujeto y representaciones sociales. Diversitas: Perspectivas en psicología, 4(2), 225-243.

Rodríguez, D. J. (2008). Buenas prácticas en el ámbito educativo y su orientación a la gestión del conocimiento. Educación Vol. XVII, N³2, 29-48.

Rodríguez-Espinosa, H., Ramírez-Gómez, C. J., \& Restrepo-Betancur, L. F. (2016). Nuevas tendencias de la extensión rural para el desarrollo de capacidades de autogestión. Ciencia y Tecnología Agropecuaria, 17(1), 31-42.

Rossato, M., \& Martínez, A. M. (2013). Desenvolvimento da subjetividade: análise de histórias de superação das dificuldades de aprendizagem. Psicologia Escolar e Educacional, 17(2), 289-298.

Sanjurjo, L. (2012). Socializar experiencias de formación en prácticas profesionales: un modo de desarrollo profesional. Praxis Educativa (Arg), 16(1), 22-32.

Silva, N. B. (2015). Relaciones entre la sociedad del conocimiento, la formación académica y la estrategia de transectorialidad gubernamental. Administración \& Desarrollo, 45(2), 149173.

Sluzki, E. (1996). La red social proposiciones generales. En: La red social: frontera de la práctica sistemática. (pp. 37-61). España: Gedisa.

Solar, M. I., \& Díaz, C. (2009). Los procesos de enseñanza-aprendizaje en el aula universitaria: una mirada desde las creencias de académicos de Trabajo Social y Periodismo. Estudios pedagógicos (Valdivia), 35(1), 181-197. 
Solís, C. A. (2015). Creencias sobre enseñanza y aprendizaje en docentes universitarios: Revisión de algunos estudios. Propósitos y Representaciones, 3(2), 227-260.

Tobón, S. (2013). Los proyectos formativos: transversalidad y desarrollo de competencias para la sociedad del conocimiento. Institutito CIFE

Torres, D. (2012). Enfoque histórico-hermenéutico: generalidades y características. Recuperado el, 21.

Vallejos Romero, A. (2012). La relevancia de la confianza institucional y la comunicación en la percepción y construcción social de riesgos. Perfiles latinoamericanos, 20(39), 151-176.

Vega-de la Cruz, L. O., \& Nieves-Julbe, A. F. (2016). Procedimiento para la Gestión de la Supervisión y Monitoreo del Control Interno. Ciencias Holguín, 22(1), 50-68.

Ventosa, V. J. (2017). Didáctica de la participación: Teoría, metodología y práctica (Vol. 67). Narcea Ediciones.

Villamar, C., María, K., \& Zambrano Alvarado, A. N. (2018). Percepciones sobre la intervención del trabajador social en el campo educativo: estudio de cuatro experiencias de profesionales del Trabajo Social en las instituciones públicas de Guayaquil.

Villarroel, V., \& Bruna, D. (2014). Reflexiones en torno a las competencias genéricas en educación superior: Un desafío pendiente. Psicoperspectivas, 13(1), 22-34.

Conflicto de intereses / Competing interests:

Los autores declaran que no incurre en conflictos de intereses.

Rol de los autores / Authors Roles:

Darwin Cruz-García. conceptualización, curación de datos, análisis formal, adquisición de fondos, investigación, metodología, administración del proyecto, recursos, software, supervisión, validación, visualización, escritura preparación del borrador original, escritura - revisar \& amp; edición.

Jimena-Ruiz: conceptualización, investigación, metodología, administración del proyecto, recursos, software, supervisión, validación, visualización, escritura - preparación del borrador original, escritura - revisar \& amp; edición.

Fuentes de financiamiento / Funding:

Los autores declaran que no recibió un fondo específico para esta investigación.

Aspectos éticos / legales; Ethics / legals:

Los autores declaran no haber incurrido en aspectos antiéticos, ni haber omitido aspectos legales en la realización de la investigación. 